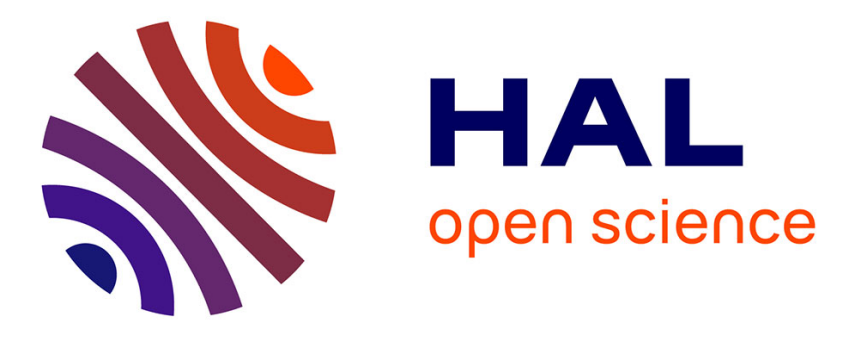

\title{
Non-human Looking Robot Arms Induce Illusion of Embodiment
}

Laura Aymerich-Franch, Damien Petit, Gowrishankar Ganesh, Abderrahmane Kheddar

\section{- To cite this version:}

Laura Aymerich-Franch, Damien Petit, Gowrishankar Ganesh, Abderrahmane Kheddar. Non-human Looking Robot Arms Induce Illusion of Embodiment. International Journal of Social Robotics, 2017, 9 (4), pp.479-490. 10.1007/s12369-017-0397-8 . lirmm-01925587

\section{HAL Id: lirmm-01925587 https://hal-lirmm.ccsd.cnrs.fr/lirmm-01925587}

Submitted on 16 Nov 2018

HAL is a multi-disciplinary open access archive for the deposit and dissemination of scientific research documents, whether they are published or not. The documents may come from teaching and research institutions in France or abroad, or from public or private research centers.
L'archive ouverte pluridisciplinaire HAL, est destinée au dépôt et à la diffusion de documents scientifiques de niveau recherche, publiés ou non, émanant des établissements d'enseignement et de recherche français ou étrangers, des laboratoires publics ou privés. 


\title{
Non-human Looking Robot Arms Induce Illusion of Embodiment
}

\author{
Laura Aymerich-Franch ${ }^{1,3}$ - Damien Petit ${ }^{1,2}$ - Gowrishankar Ganesh $^{1,2}$. \\ Abderrahmane Kheddar ${ }^{1,2}$
}

Accepted: 5 February 2017

(C) Springer Science+Business Media Dordrecht 2017

\begin{abstract}
We examine whether non-human looking humanoid robot arms can be perceived as part of one's own body. In two subsequent experiments, participants experienced high levels of embodiment of a robotic arm that had a blue end effector with no fingers (Experiment 1) and of a robotic arm that ended with a gripper (Experiment 2) when it was stroked synchronously with the real arm. Levels of embodiment were significantly higher than the corresponding asynchronous condition and similar to those reported for a human-looking arm. Additionally, we found that visuomovement synchronization also induced embodiment of the robot arm and that embodiment was even partially maintained when the robot hand was covered with a blue plastic cover. We conclude that humans are able to experience a strong sense of embodiment towards non-human looking robot arms. The results have important implications for the domains related to robotic embodiment.
\end{abstract}

Electronic supplementary material The online version of this article (doi:10.1007/s12369-017-0397-8) contains supplementary material, which is available to authorized users.

$\triangle$ Laura Aymerich-Franch

laura.aymerich@gmail.com

1 CNRS-AIST Joint Robotics Laboratory (JRL), UMI3218/RL, National Institute of Advanced Industrial Science and Technology (AIST), Central 1, 1-1-1 Umezono, Tsukuba, Ibaraki 305-8560, Japan

2 CNRS-UM LIRMM, Interactive Digital Human Group, UMR5506, Montpellier, France

3 National Institute of Advanced Industrial Science and Technology (AIST), Central 1, 1-1-1 Umezono, Tsukuba, Ibaraki 305-8560, Japan
Keywords Humanoid robot embodiment · Non-human looking robot · Body ownership illusion · Rubber-hand illusion

\section{Introduction}

Where does the limit lie regarding the brain's capacity to integrate a non-human looking arm as part of one's own body? In the rubber hand illusion (RHI), participants have the sensation that a human-looking rubber hand becomes part of their body when it is stroked synchronously with their real hand, which stays out of sight [1]. On the other hand, the illusion of embodiment is not perceived when the physical characteristics of the fake hand are very different to those of the real hand, such as when a wooden stick is used instead of a rubber hand [2]. Regardless of that, participants can still experience the illusion of touch on a non-hand object like a box [3] or a table [4] when there is prior onset of the basic RHI [3,4].

The acceptance of a fake limb as part of the body by the brain depends on several physical and spatial factors. Regarding physical features, it is known that resemblance of the limb or body to a human body contributes to increase the sense of embodiment [2,5]. However, factors such as color [6] or skin luminance [7] do not affect the illusion of embodiment of fake hands. Interestingly, regarding size, previous work shows that while human participants experience the embodiment illusion of a rubber hand larger than the real one, they do not experience the illusion when a rubber hand smaller than the real one is used [8]. Regarding spatial features, previous research has found that the illusion of embodiment of a rubber hand does not occur when the posture of the rubber hand is spatially incongruent with respect to the real hand $[2,9,10]$ or when the rubber hand is located outside the participant's peri-personal space [11]. 


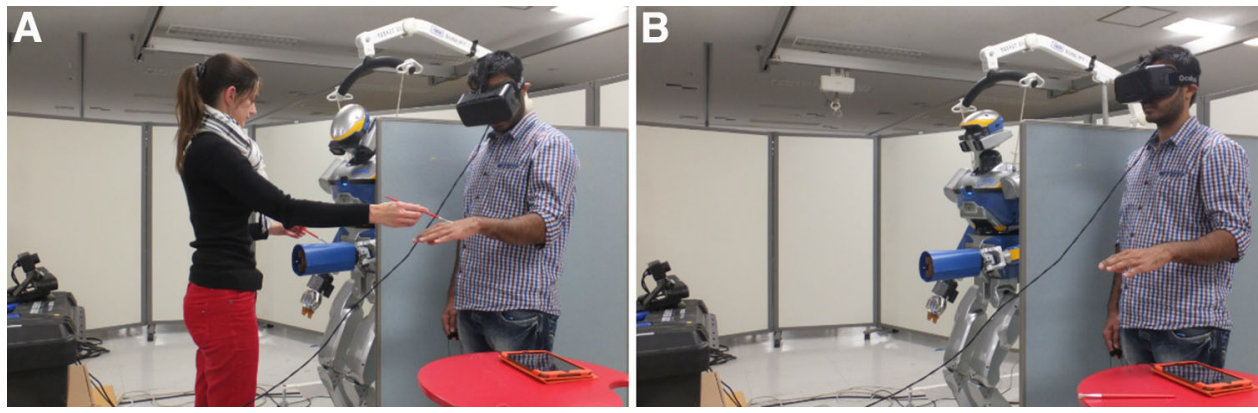

Fig. 1 Humanoid robot embodiment set-up used in both experiments. The participant wears an HMD that provides vision from the robot's perspective and is able to control the robot's head movement. The illusion is either induced by visuo-tactile synchronization (a) or visuo-movement synchronization (b)

In the particular domain of robots, previous works have reported body ownership transfer towards teleoperated android arms or robot arms either using visuo-movement synchrony [12,13] or brain-computer interface [14], but have only utilized arms with high resemblance to humans in terms of shape (i.e. a hand with five fingers) [12] and texture [3,13]. On the other hand, studies in virtual reality suggest that users are able to identify with avatars even when they present different visual characteristics than their real selves provided that there exists visuo-movement correlation between the human body movement and the avatar movement [15-17]. Thus, it is not clear where the limit lies regarding humans' capacity to integrate non-human looking robot arms as part of their body.

In order to explore this question further, we conducted two experiments utilizing our humanoid robot embodiment set-up (Fig. 1a, b) in which we induced the illusion of embodiment in two different types of humanoid arms by visuo-tactile synchronization (Fig. 1a), following the classic rubber hand illusion experiment [1]. Specifically, the first experiment examined embodiment of a non-human looking robot arm made of metal and plastic which ended with a blue hand (end effector) with no fingers (Fig. 2a) and the second experiment examined embodiment of a robot arm which ended with a metal gripper (Fig. 2b). In addition, previous research suggests that movement synchronization between the real and the fake body or body part are also able to induce the illusion of embodiment in fake or robotic limbs and bodies when they resemble human shape $[12,13]$. Thus, our experiments also examined whether sense of embodiment of the robot arm is also achieved when visuo-movement synchronization (i.e. arm movement synchronization between the human arm and the humanoid arm) is used to induce the illusion (Fig. 1b). Finally, since previous research suggests that embodiment illusion does not occur when an object such a box is used for the illusion [3], the second experiment additionally explored whether embodiment takes place when the robot hand is covered by a plastic cover (Fig. 2c).
A novelty in our study, compared to previous rubber-hand illusion related experiments, is that we utilize a whole body embodiment set-up $[18,19]$. In our setup participants embody the humanoid robot using a head-mounted display (HMD) that provides vision of the arm from the robot's perspective (Video S1) and also control the robot's head movement (Fig. 1a, b). This enabled us to present an artificial limb (arm) to the participants at the same location as their real limb, a feature we believe is key to the various results we observed in this study. The robot avatar setup uses similar principles to the ones used in virtual reality to achieve sense of embodiment of the avatar body (i.e. first person perspective from the avatar body and visuo-movement synchronization of the user and the avatar head).

\section{Method}

\subsection{Participants}

In total, 31 participants took part in the study. Twelve participants of different nationalities (six males and six females), aged 21-43 ( $\mathrm{M}=26.67, \mathrm{SD}=8.04)$, participated in the first experiment and nineteen participants also of different nationalities (thirteen females and six males), aged 23-44 $(\mathrm{M}=31.77, \mathrm{SD}=6.02)$, participated in the second experiment. One of the participants was left-handed in each study and the rest were right-handed. Participants were naïve to the purpose of the experiment and received 1500JPY (Japanese yen) to participate. They were recruited through a call for volunteers in a webpage created ad hoc for the experiment, which was allocated on a social network. Working in the Robotics or Neuroscience fields was used as exclusion criteria. A pretest was conducted for each study with five (Experiment 1) and three (Experiment 2) intern master students of the research laboratory in which the study was conducted. The study was carried out with ethical approval of the National Institute of Advanced Industrial Science and Technology (AIST) in Tsukuba, Japan. 

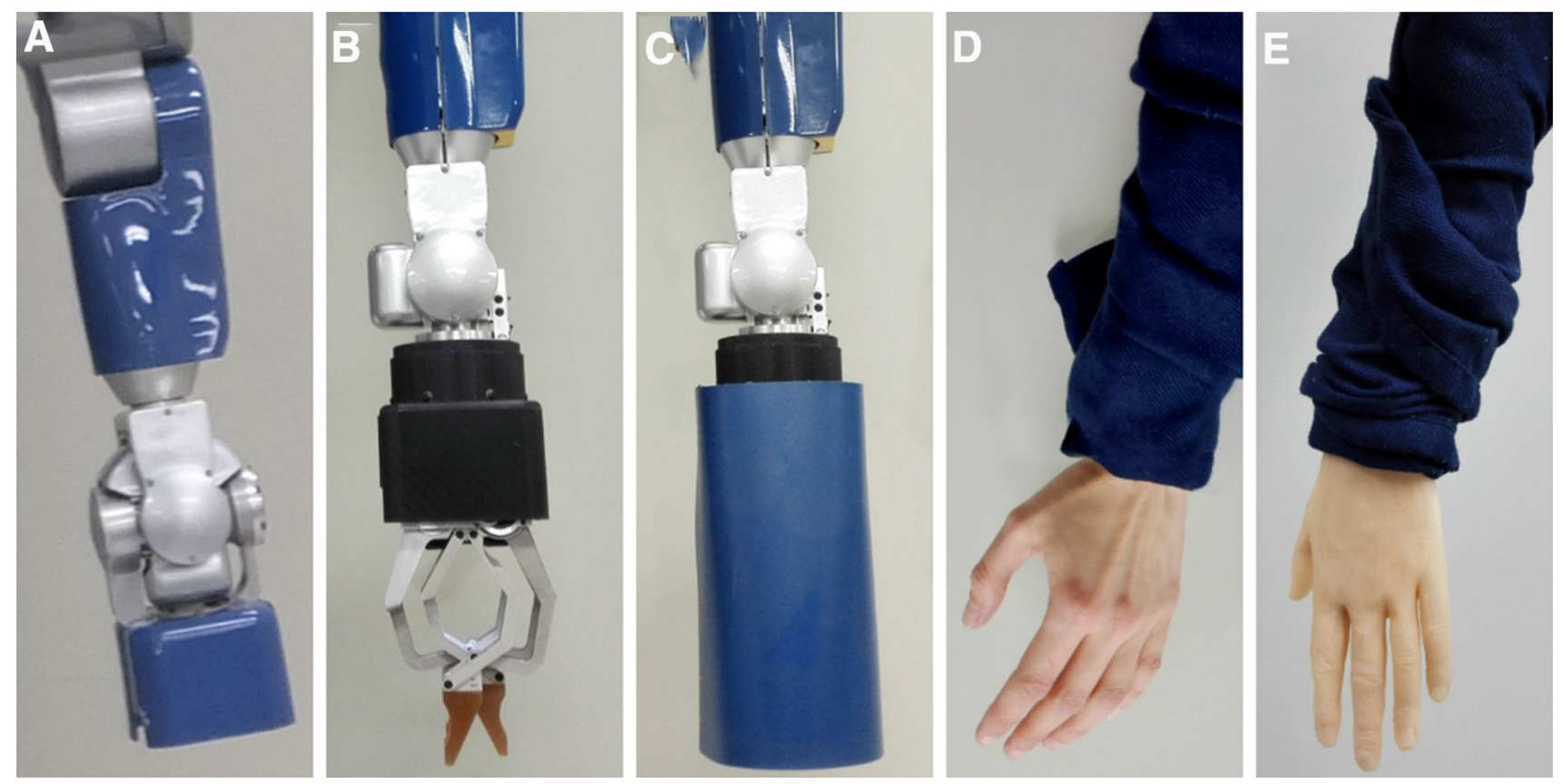

Fig. 2 Blue hand with no fingers (A), gripper (B), hand covered with a blue plastic cover (C), human hand (D), human-looking rubber hand (E) used in the experiments A and D were used in Experiment 1 while B, C, and D were used in Experiment 2

\subsection{Apparatus and Material}

A human size humanoid robot HRP-2 [20] was used to create embodiment in the robot arm conditions. The material of the robot arm was composed of plastic and metal of blue and silver colors. Each arm had seven degrees of freedom. A RGB-D camera (Asus XtionPRO live) was mounted on the robot's head which sent visual feedback to an Oculus Rift HMD worn by the user (Fig. 1a, b). Sensors integrated in the HMD allowed the tracking of the user's head motion in order to control the robot's head. A second RGB-D camera (similar to the previous one) was used externally to track the hand motion of the user in the robotic arm visuo-movement conditions (Fig. 1b). OpenNI, a framework to track body and hand motion, was used to track the user's hand position. In our experiment, only the user's hand position was tracked. The hand tracker was initialized after detecting a waving motion of the user's hand. Once the hand was tracked, participants were asked to place their hand in a position which corresponded to the robot's hand position (See Procedure). Next, the user hand motion was mapped on the robot hand (teleoperation mode). In order to control the robot arm and head, the Stack-of-Task (SoT) controller was used [21], which took as input the hand position provided by OpenNI as well as the user's head orientation provided by the HMD. In the SoT, the tasks are defined as state error vectors in the sensory space, and projected in the robot joint space with the robot (pseudo)inverse kinematic Jacobian. The Robot Operating System (ROS) was used to integrate the HMD, the robot's camera, the SoT, and the human hand tracking. In Experiment 1, the robot had a blue square ending at the place of the hand, which had no fingers (Fig. 2a, Video S1). In Experiment 2, the robot had a metal gripper with three fingers (Fig. 2b, Video S1). For the cover condition in Experiment 2, a blue cylinder composed of hard plastic was used to cover the robot arm (Fig. 2c, Video S1).

For the human arm conditions, we used a medium big sized bare arm corresponding to a female researcher (Fig. 2d, Video S1) in Experiment 1 and a rubber hand (Fig. 2e, Video S1) in Experiment 2. In those conditions, the robot arm was lowered and left aligned to its body and substituted by the human arm, so that the participants were able to see a human arm when they looked down through the HMD (Fig. 1a, b, Video S1). In order to keep the human arm in Experiment 1 neutral (Fig. 2d), it did not have any visible clothes or jewelry. The nails were cut short and did not have any nail polish.

\subsection{Procedure}

After reading and signing the consent form, participants were placed next to a panel. The humanoid robot was at the other side of the panel and was not visible to them (Fig. 1a, b). Participants then wore an Oculus Rift visor which was used to display real-time video feedback from the camera located at the forefront of the robot's head, right above its eyes. Once the participants wore the visor they were able to control the robot's head movement and visualize what the robot was seeing in real-time (Video S1). The participants were then 
told to place their arm (left, or right arm, depending on the condition and experiment) creating a $90^{\circ}$ angle between the arm and the forearm, with the elbow next to the hip, and to leave their hand opened. This specific arm position was similar to the robot's arm posture so that users observed the robot arm coinciding with their own arm position. Also, they were told to look at the robot's arm through the visor. This position was valid for all experimental conditions. After this, the first trial started. For the human arm synchronous and asynchronous condition, the robot's arm was removed from the vision of the participant and either one of the researchers put her real arm on the place of the robot's arm while the other researcher stroked the researcher's arm (Experiment 1) or a rubber arm was positioned at the place of the robot arm (Experiment 2). For the visuo-tactile synchronous and asynchronous conditions, a researcher stroked the robot's or the human-looking arm and the participant's arm either synchronously or asynchronously, with two small paintbrushes. For the robot visuo-movement synchronization condition, participants were told to look front and wave. A camera was placed in front of the participant to track the arm movement. Participants could not see the camera because they wore the visor. Then, they were asked to place their real arm creating a $90^{\circ}$ angle and looking at the robotic arm through the visor. After that, participants were able to move their arm and the robot's arm responded with the same movement. For the asynchronous robot arm control conditions, the camera that tracked the participant's movement was flipped and faced one of the researchers, who performed the control instead. In Experiment 1, after $90 \mathrm{~s}$ of either stroking or arm movement, the participants were verbally asked the three questions corresponding to the embodiment questionnaire (see Measures) by another researcher while they kept observing the arm, and responded the answers out loud. In Experiment 2, before asking the questions, one of the experimenters passed his hands around the fake limb for $15 \mathrm{~s}$ in order to examine potential changes on skin conductance. GSR data was recorded during this period of time. After that, the embodiment questionnaire was asked while the participant kept looking at the arm. Then, they relaxed and moved their arm for few seconds and continued to the next condition. The participants looked front every time the setup was modified so that they were not able to see the modifications taking place among the different conditions. Participants were also requested to look front for few seconds between the synchronous and the corresponding asynchronous condition even if the setup was not modified. In Experiment 1, after completing all conditions, participants started over with the opposite arm. In Experiment 2, participants only completed each condition once, with the left arm. At the end of Experiment 1 participants answered an open-ended question while in Experiment 2 a short-interview was conducted by one of the experimenters (see Measures). Finally, they were thanked and paid for their participation.

\subsection{Measures}

\subsubsection{Embodiment Questionnaire}

As a working definition, we understand embodiment as the illusion of adopting an artificial body or body part as part of the own body. Although there is no broad consensus, embodiment can be considered to consist of three subcomponents: the sense of body ownership, the sense of self-location, and the sense of agency $[22,23]$. Body-ownership is the sense of owning a body or body part. Self-location is a determinate volume in space in which one feels to be located, normally localized within the bodily boundaries [24]. Finally, agency is "global motor control, including the subjective experience of action, control, intention, motor selection and the conscious experience of will" [24]. For Experiment 1, a short-form embodiment questionnaire was designed to measure arm embodiment of the embodied arm based on other studies [22,23]. Also following previous studies [17], the questionnaire was specifically designed to be responded in situ in order to minimize the effect of recall bias [25]. Brief versions of self-rated questionnaires such as the Mini-Social Phobia Inventory [26], consisting of only three items, demonstrate that shortened versions of assessment instruments are as efficient [26], and demonstrate reliability and validity [27] similar to the corresponding longer versions. The questionnaire has also been implemented elsewhere [18,28]. It consisted of three questions ("Appendix 1" section), one for each of the sub-dimensions of embodiment: body ownership, self-location, and agency [22]. The questionnaire was designed so that the same questions were suitable to measure human arm embodiment and robot arm embodiment. The scale obtained an average reliability of $\alpha=.94$, $\mathrm{KMO}=.605$, and Bartlett's test of Sphericity $=.002$. For Experiment 2, we adapted the embodiment questionnaire from [23] to measure the sense of embodiment to the robot's arm. The questionnaire consisted of nine items ("Appendix 1 " section) which were rated on a 7-point scale that ranged from (1) not at all to (7) very strongly. The scale consisted of the same sub-dimensions described for the previous questionnaire: sense of body ownership (five items), sense of self-location (two items), and sense of agency (two items). Average reliability of the scale was $\alpha=.93$.

\subsubsection{Demographic Measures}

At the end of the experiment participants completed their age, gender, and whether they were right or left-handed in a PC-based questionnaire. 


\subsubsection{Galvanic Skin Response (GSR)}

In Experiment 2 we measured skin conductance which was recorded using a Mindfield Biosystems eSense Skin Response device and processed using MatLab. Skin conductance was measured with two electrodes attached to the middle and index finger of the right hand. Sampling frequency of the device was $10 \mathrm{~Hz}$ (10 values per second) with a resolution of 18 bit, with five values per second saved for exported data to a CVS file. The participants wore the electrodes throughout the experiment and skin conductance was recorded from the beginning to the end of each experimental condition. We examined a period of $15 \mathrm{~s}$ occurring right after the $90 \mathrm{~s}$ of visuo-tactile or visuo-motor stimulation during which one of the researchers passed his hands around the embodied arm while the participants looked at it.

\subsubsection{Open-Ended Question/Interview After the Experiment}

In Experiment 1, an open-ended question after completing the demographic measures asked participants to share their thoughts about the experience by explaining in which condition the feeling that the arm was their own arm was stronger. In Experiment 2, a brief interview was conducted with each participant at the end of the experiment to collect qualitative data about the sensations experienced in each experimental condition in more detail ("Appendix 1" section).

\subsubsection{Manipulation Check}

At the end of each condition participants were asked whether the touch and vision were happening at the same time or at different times or whether the robot arm responded to their movements. One participant in Experiment 2 was discarded because she repeatedly gave higher scores for all the asynchronous conditions compared to the corresponding synchronous conditions.

\section{Experiment 1}

In the first experiment, we examined the sense of embodiment of a robotic arm that had a blue hand with no fingers (Fig. 2a, Video S1). The sense of embodiment was induced by visuotactile synchronization, following the rubber-hand illusion paradigm [1]. Additionally, a condition was added in which the embodiment illusion of the robot arm was induced by visuo-movement synchronization. Specifically, we expected that embodiment would be significantly higher in the robotic arm visuo-tactile synchronous condition $\left(\mathrm{H}_{1 \mathrm{a}}\right)$ as well as in the visuo-movement synchronous condition $\left(\mathrm{H}_{1 b}\right)$, compared to the visuo-tactile asynchronous condition. Based on a recent proposal that the functional characteristics of a limb are more important than the physical features for embodiment [30], we expected that embodiment in the robotic arm visuo-tactile synchronous conditions would be similar to that obtained in the synchronous human arm condition $\left(\mathrm{H}_{2 \mathrm{a}}\right)$. Similarly, we expected that embodiment in the robotic arm visuo-movement synchronous conditions would be similar to that obtained in the synchronous human arm condition $\left(\mathrm{H}_{2 b}\right)$. Based on previous findings which suggest that embodiment of a fake limb can also be induced using visuo-movement correlation [29], we expected that levels of embodiment in the robotic arm visuo-tactile synchronous condition and embodiment in the robotic arm visuo-movement synchronous condition would be similar $\left(\mathrm{H}_{2 \mathrm{c}}\right)$.

In order to examine these hypotheses, a within-subject experiment was conducted in which participants experienced a total of five conditions ("Appendix 2" section):

$$
\begin{aligned}
& {[(\text { robot, human }) \times(\text { sync., async. visuo-tactile })]} \\
& \quad+(\text { robot visuo-movement sync. })
\end{aligned}
$$

Participants repeated each condition twice: once for the left arm and once for the right arm. Half of the participants started with the left arm and half of them started with the right arm and went through the five trials before switching to the opposite arm. We grouped the synchronous with its corresponding asynchronous condition and randomized the order between them and among the groups of conditions such that each participant experienced the conditions in a different order but synchronous and asynchronous conditions of different types of arms were not mixed among them.

\subsection{Results}

Descriptive statistics for overall embodiment as well as for each subcomponent are reported by experimental condition in Table 1. For each condition, we averaged the results of the left and the right arm, which were not significantly different ( $p>.05$ in all group comparisons). Given the relatively small size of the sample, nonparametric tests were conducted. Friedman test yielded a statistically significant difference among groups $\chi^{2}(4)=26.000, p<.001$. Wilcoxon signed-rank tests were conducted with Bonferroni correction (significance level applied at $p=.008$ ) to further examine the differences between conditions and respond to the hypotheses. The sense of embodiment of a human arm induced by visuo-tactile synchronization was significantly higher in the synchronous condition, compared to the asynchronous condition $(Z=-2.984, p=.001$, onetailed), which validated the experimental design. Regarding the first hypothesis, the sense of embodiment of a robot arm induced by visuo-tactile synchronization was significantly higher in the synchronous condition, compared to the asynchronous condition $(Z=-2.944, p=.003$, two-tailed), 
Table 1 Mean (SD) for overall embodiment and its subcomponents

\begin{tabular}{lllll}
\hline & Overall embodiment & Ownership & Self-location & Agency \\
\hline Human sync. & $5.11(1.06)$ & $5.5(.96)$ & $5.75(.69)$ & $5.43(1.13)$ \\
Human async. & $3.76(1.37)$ & $3.78(1.38)$ & $4(1.15)$ & $3.57(1.61)$ \\
Robot sync. & $5.33(1.04)$ & $5.78(.91)$ & $5.64(1.11)$ & $6.14(.89)$ \\
Robot async. & $3.87(1.44)$ & $4.14(2.25)$ & $4.5(1.44)$ & $4.5(1.25)$ \\
Robot visuo-mov & $5.29(1.02)$ & $5.36(1.18)$ & $5.07(1.36)$ & $6.14(.94)$ \\
\hline
\end{tabular}

which supports $\mathrm{H}_{1 \mathrm{a}}$. Also, the sense of embodiment of a robot arm induced by visuo-movement synchronization was significantly different than the asynchronous robot arm condition ( $Z=-3.062, p=.002$, two-tailed), which supports $\mathrm{H}_{1 \mathrm{~b}}$. Regarding the second hypothesis, the sense of embodiment of a robot arm induced by visuo-tactile synchronization and the sense of embodiment of a human arm induced by visuo-tactile synchronization were not significantly different ( $Z=-1.070, p=.285$, two-tailed), which supports $\mathrm{H}_{2 \mathrm{a}}$. Levels of embodiment were equally high for the human and for the robot arm (See Table 1). Also, the sense of embodiment of a robot arm induced by visuo-movement synchronization was not significantly different to the sense of embodiment obtained in the human arm synchronous condition $(Z=-.409, p=.683$, two-tailed), which supports $\mathrm{H}_{2 \mathrm{~b}}$, or to the sense of embodiment of a robot arm induced by visuo-tactile synchronization $(Z=-.544, p=.579$, twotailed), which supports $\mathrm{H}_{2 \mathrm{c}}$. Levels of embodiment obtained in the visuo-movement synchronization condition were very similar to those obtained in the synchronous visuo-tactile human and robot conditions.

Finally, the qualitative data resulting from the open questions supported the results obtained in the questionnaire. In particular, participants reported a strong feeling that the robot arm was part of their body: "The feeling that the robot arm was part of my own body was strong... the feeling when I saw the real arm and the feeling when I saw the robot arm was pretty much the same because I felt the robot arm as my own" or "the robot arm was very similar to my arm... the feeling was very much the same feeling to when I saw the real arm". In addition, the qualitative data revealed that while some participants felt stronger embodiment when they controlled the movement of the robot: "it was stronger when I controlled the movement than when I got the paintbrush stroke", some others felt stronger sense of embodiment when they received visuo-tactile synchronization: "the feeling that it was my own arm was the strongest when I saw the robot's arm and got the paintbrush stroke which was synchronized".

\section{Experiment 2}

In Experiment 2, we examined embodiment of a robotic arm with a gripper (Fig. 2b, Video S1). To additionally explore where the limit lies regarding the brain capacity to embody external entities, this experiment also examined whether the sense of embodiment was maintained when the robot arm was not visible and was covered by a blue plastic cover. Experiment 2 also improved some aspects of the methodology used in Experiment 1. Specifically, skin conductance was included as a measure to provide objective physiological evidence for the illusion. The human arm was substituted for a rubber hand following the method used in most RHI experiments [1]. Also, we adopted a more validated questionnaire of embodiment [23] and added a brief interview with the participant at the end of the experience instead of the open question to collect the qualitative aspects of the experience in a more interactive and detailed manner ("Appendix 1" section). Finally, we included an asynchronous visuo-movement condition in the design to compare it with the corresponding synchronous condition.

Specifically, for this experiment, participants experienced the embodiment illusion of a gripper (Fig. 2b, Video S1) and a human-looking arm (Fig. 2e, Video S1). We expected that embodiment would be significantly higher in the gripper visuo-tactile synchronous condition compared to the corresponding asynchronous condition $\left(\mathrm{H}_{1}\right)$. Also, we expected that embodiment of the gripper would be similar to that obtained in the synchronous human-like rubber hand condition $\left(\mathrm{H}_{2}\right)$.

Additionally, we examined whether embodiment was present when the arm was not visible (i.e. covered by a blue cover) and it was stimulated either by visuo-tactile $\left(\mathrm{RQ}_{\mathrm{a}}\right)$ or visuo-movement synchronization $\left(\mathrm{RQ}_{1 \mathrm{~b}}\right)$. We chose an object which possessed drastically different visual properties from a human arm, specifically, in terms of shape, material, and color (Fig. 2c, Video S1). In the cover conditions, the illusion was induced by synchronous or asynchronous visuotactile or visuo-movement synchronization.

In order to test the hypotheses and research questions, a within-subject design was conducted in which participants experienced a total of eight conditions ("Appendix 2" section):

\footnotetext{
[(robot, human $) \times($ sync., async. visuo-tactile $)]$

$+[($ visuo-tactile, visuo-movement cover $) \times($ sync., async. $)]$
} 
Table 2 Mean (SD) for overall embodiment and its subcomponents

\begin{tabular}{llllll}
\hline & Overall embodiment & Ownership & Self-location & Agency & GSR \\
\hline Human sync. & $5.13(1.35)$ & $5.27(1.39)$ & $5.17(1.55)$ & $4.97(1.45)$ & $3.23(1.48)$ \\
Human async. & $4.33(1.05)$ & $4.14(1.38)$ & $4.61(1.09)$ & $4.25(1.34)$ & $2.98(1.3)$ \\
Gripper sync. & $4.56(1.41)$ & $4.31(1.45)$ & $4.82(1.51)$ & $4.56(1.63)$ & $3.34(1.41)$ \\
Gripper async. & $3.35(1.6)$ & $2.69(1.66)$ & $3.79(1.72)$ & $3.56(1.99)$ & $3.13(1.28)$ \\
Cover visuo-tact. sync. & $4.97(1.33)$ & $4.6(1.48)$ & $5.22(1.32)$ & $5.08(1.62)$ & $2.90(1.4)$ \\
Cover visuo-tact. async. & $3.59(1.56)$ & $3.12(1.5)$ & $4.03(1.87)$ & $3.61(2.09)$ & $2.93(1.37)$ \\
Cover visuo-mov. sync. & $5.01(1.12)$ & $4.51(1.39)$ & $4.88(1.24)$ & $5.65(1.32)$ & $3.19(1.3)$ \\
Cover visuo-mov. async. & $2.76(1.78)$ & $2.42(1.65)$ & $2.88(1.85)$ & $2.97(2.33)$ & $3.18(1.39)$ \\
\hline
\end{tabular}

Since no significant differences were found between the two arms in Experiment 1 (see Results), in Experiment 2 participants tried each condition only once with the left arm (for being the one used in the classical RHI experiment [1]). We followed the same procedure for randomizing the conditions than in the previous experiment (See Experiment 1).

\subsection{Results}

Descriptive statistics for overall embodiment (resulting from the average of the three subcomponents: ownership, selflocation, and agency) as well as for each subcomponent by experimental condition are reported in Table 2. Some groups of data (i.e. the data in the cover visuo-movement asynchronous condition and in the rubber arm synchronous conditions) did not reach normal distribution according to the Shapiro-Wilk test. Thus, non-parametric tests were used for the statistical analysis. Friedman test yielded a statistically significant difference among groups $\chi^{2}(7)=$ $54.715, p<.001$. Wilcoxon signed-rank tests with Bonferroni correction (significance level applied at $p=.005$ ) were conducted in order to further compare the different conditions between them and answer the hypotheses and research questions. It was found that the sense of embodiment was significantly higher in the human-looking rubber $\operatorname{arm}(Z=-2.557, p=.005$, one-tailed) compared to the corresponding asynchronous version, which validated the experimental design. Also, embodiment of the gripper arm ( $Z=-3.364, p=.001$, two-tailed) was significantly higher in the synchronous than in the corresponding asynchronous version, which supports $H_{1}$. Additionally, in response to $R_{1}$ and $\mathrm{RQ}_{2}$, embodiment in the cover arm induced by visuotactile synchronization ( $Z=-3.724, p<.001$, two-tailed), and the cover arm induced by visuo-movement synchronization $(Z=-3.527, p<.001$, two-tailed) were also significantly higher compared to their corresponding asynchronous versions. Also, levels of embodiment obtained in all the visuo-tactile and visuo-movement synchronous conditions were very similar among them (see Table 2). In support to $\mathrm{H}_{2}$, the sense of embodiment was not significantly different in the rubber-hand compared to the gripper ( $Z=-.1 .065, p=.287$, two-tailed). Again regarding $\mathrm{RQ}_{1}$ and $\mathrm{RQ}_{2}$, embodiment was also not significantly different in the rubber-hand compared the cover arm induced by visuo tactile synchronization ( $Z=-.024, p=.981$, two-tailed), or the cover arm induced by visuo-movement synchronization ( $Z=-.355, p=.723$, two-tailed). Also, levels of embodiment in the gripper were not significantly different than in the cover arm induced by visuo tactile synchronization $(Z=-.848, p=.397$, two-tailed) or in the cover arm induced by visuo-movement synchronization $(Z=-1.221$, $p=.222$, two-tailed). Levels of embodiment between the cover arm induced by visuo-movement or visuo-tactile synchronization were also not significantly different between them $(Z=-.398, p=.691$, two-tailed).

In line with the results obtained in the questionnaire, participants displayed significantly greater GSR responses in the synchronous visuo-tactile human-like rubber arm condition compared to the corresponding asynchronous condition $(t(14)=1.929, p=.044$, one-way). Also, GSR approached significance in the synchronous gripper arm condition compared to the corresponding asynchronous condition $(t(14)=1.684, p=.057$, one-way). Additionally, GSR levels in the synchronous visuo-tactile human-like arm and synchronous visuo-tactile gripper arm conditions were not significantly different between them $(t(14)=$ $-.731, p=.477$, two-way). The arm covered conditions did not reach significance. Cover visuo-movement synchronous and asynchronous conditions were not significantly different between them $(t(14)=.087, p=.465$, one-way) due to that in both conditions GSR levels were high (see Table 2). In fact, synchronous visuo-tactile human-like and synchronous visuo-movement cover condition were not significantly different between them $(t(14)=.155, p=.879$, two-way). Also, cover visuo-tactile synchronous and asynchronous conditions were not significantly different between them $(t(14)=-.381, p=.646$, one-way). In this case, synchronous visuo-tactile human-like was significantly higher 
Fig. 3 Skin conductance responses for Experiment 2: $\mathrm{M}(\mathrm{SD})$ values resulting from subtracting the responses obtained in the asynchronous condition to the corresponding synchronous condition

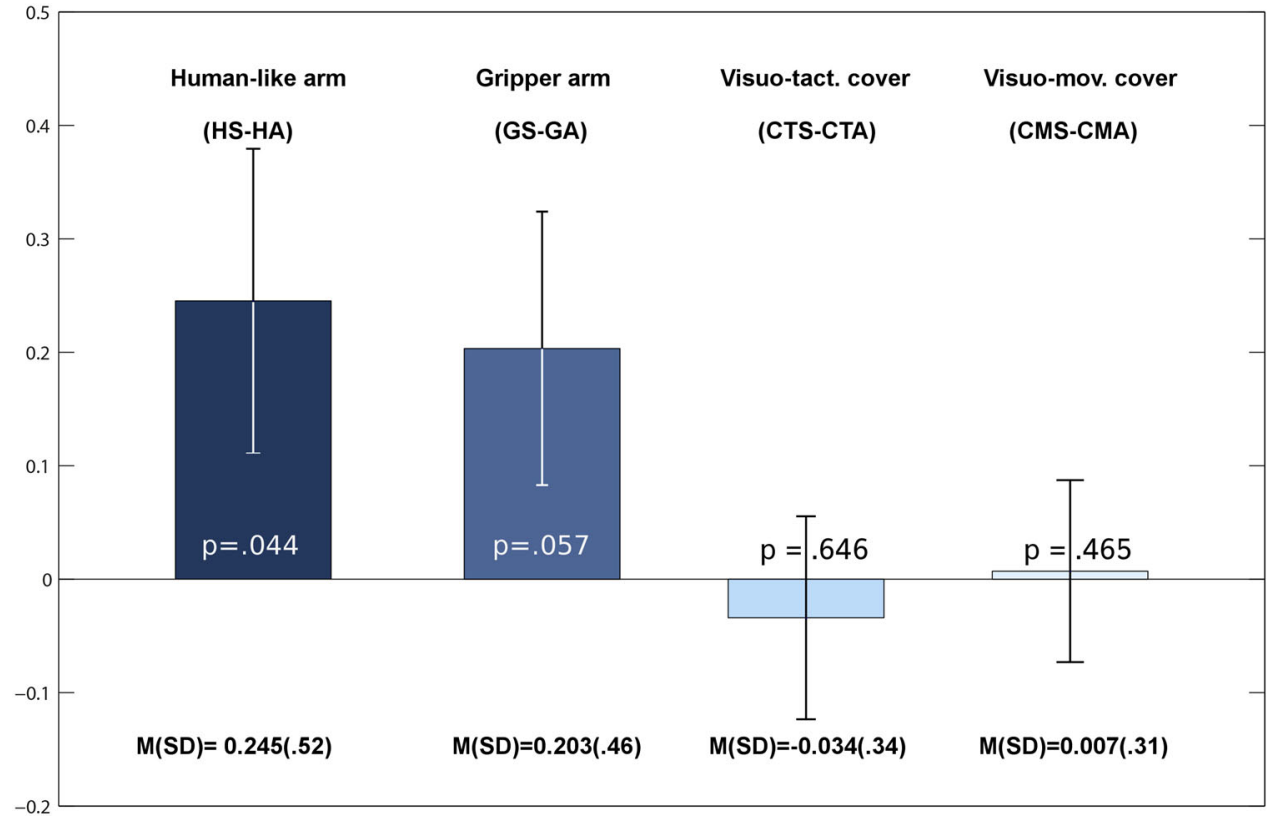

than synchronous visuo-tactile cover arm condition $(t(14)=$ $3.281, p=.005$, two-way). Table 2 shows GSR mean and SD by condition (Fig. 3).

Finally, the qualitative data resulting from the interviews after the experiment pointed in the same direction than the reported quantitative measures. In particular, the comments from participants support that the synchronous conditions generated stronger embodiment than the asynchronous conditions both in the human-like and in the robot arms and that embodiment in the robot arms was very similar to that experienced with the human-like arm: "when the hand touched the arm I felt a very strong sensation (that it was touching me) in all the synchronous conditions". However, there was no unified response regarding which of the synchronous conditions (including human and robot conditions) induced the strongest illusion. Also, some participants experienced real sensations in their hands such as heat or tickling when the researcher passed his hand around the robot or the humanlooking hand: "I felt tickling during the hand passing with the metal hand" or "I felt warm in the left hand and arm when you touched the arm". The fact that the sense of embodiment was really perceived is also evident in sentences such as "It was a little bit scary and strange when the hands touched me" (i.e. the hands never touched "me", that is, the real person, but only the embodied limb) or "I felt the robot's arms as if they were a prosthesis". For the arm covered condition, some participants felt stronger embodiment when they controlled the movement of the robot: "I feel more embodied when I moved the arm. I felt like it was my real arm. The feeling was increasing from nothing at all to believe that I was the real owner", while some others felt stronger sense of embodiment when they received visuo-tactile synchronization.

\section{Discussion}

Our study manipulated the specific variables of physical appearance and technique to induce embodiment using a wide range of combinations in order to explore the sense of embodiment of a non-human looking robot arm. Regarding technique, the experiments included the two main paradigms generally employed to generate sense of embodiment in fake bodies and body-parts: visuo-tactile synchronization, which is the technique used in the studies related to the classic rubber-hand illusion paradigm [1], and visuo-movement synchronization, which is the technique generally used in virtual reality to induce sense of avatar embodiment [22]. Regarding physical appearance, two different types of non-human looking humanoid robot arms were tested: a blue hand with no fingers, and a gripper. The blue arm was selected because it presents a series of characteristics that are drastically different from the human arm in terms of material, color, texture, and shape. The gripper was selected because it represents a type of end effector generally employed in non-human looking humanoid robots. Furthermore, we considered these two types of arms because they differed in having fingers or not, which we believe is a key functional feature defining one's hand and arm [30]. In addition to these two types of arms, the cover condition was included to assess whether a robot arm and a non-arm object in the place of the arm produced differences in the sense of embodiment.

The response to the rubber hand illusion is known to greatly vary across individuals, and only about $70 \%$ of individuals are known to experience it $[9,11,31]$. We therefore chose to use a within-subjects design over a between-subjects design for its strength in accounting for individual differences 
in subject responses [32,33]. Furthermore, it is generally assumed that within-subjects designs are more powerful (more likely to detect differences between conditions if they exist) than between-subjects designs [32,34]. However, within subject designs can be affected by learning or interference effects. We used a random condition presentation order across participants to attenuate any learning effects $[32,33]$. To attenuate any interference effects, we asked participants to relax and move their arm between conditions. The significant differences between the synchronous and asynchronous conditions validate our experimental design.

The results of this study demonstrate that people are able to experience a similar sense of embodiment of a non-human looking humanoid robot arm than that experienced for a human-looking arm in a classic rubber-hand illusion experiment [1]. In particular, we show that participants are able to experience a sense of embodiment towards a non-human looking metal and plastic robot arm with a blue hand with no fingers and towards a non-human looking metal and plastic robot arm with a metal gripper. Additionally, we demonstrate that embodiment towards the non-human looking robot arm is also perceived when visuo-movement synchronization is used instead of visuo-tactile stimulation. Finally, we show that embodiment is at least partially maintained when the robot arm is covered, specifically, by a blue plastic cover (“Appendix 3" section).

Our embodiment set-up $[18,19,28]$, which used an HMD that provided first person perspective from the robot's body and robot's head movement control by the participant, enabled us to provide participants with a visual illusion where the robot arm was in the same location as the participant's real arm (Video S1), in turn enabling spatial coincidence between the visually perceived touch and the felt touch. We believe that this aspect of the experimental design, which differs from the traditional RHI experiment in which the real arm is in a different location than the rubber hand, contributed to enhance the sense of embodiment towards the robot's arm. We also speculate that the enhanced embodiment setup might explain why participants felt embodiment even when the arm was covered with a blue plastic cover, which contradicts previous experiments using the traditional rubber-hand illusion setup [2]. On the other hand, the embodiment of the gripper was expected by us as we believe that the functional features like fingers are more important for embodiment than the actual physical appearance of the fingers [30].

Indeed, some participants experienced high sense of embodiment even in the asynchronous conditions. A participant explained that "When you performed the asynchronous move, I felt like it was my brain problem, something like I was drunk so my brain was working slowly... not the robot problem." Previous experiments that have used a similar method to induce embodiment in a humanoid robot have also obtained high ratings of embodiment, which remain even when the robot is not controlled by the human operator [18]. Thus, it would be interesting to examine if the illusion of embodiment remains when a non-human looking robot arm is detached from the robot. We believe that the fact that the arm is attached to the robot and visualized in first person perspective from the viewpoint of the humanoid robot contributes to conceptualize it as an "arm" and thus develop the functions associated to this limb, which in turn contributes to integrate it as part of the body [30]. On the other hand, if the same arm was seen on a table detached from the robot, it might be perceived as an "object", with no connection with the body.

We note here that regarding the cover conditions, whereas the results of the questionnaire indicate that embodiment was significantly higher in the synchronous than asynchronous conditions and similar to the human-like arm synchronous condition, the results of the GSR data did not reach significance. A potential explanation for this might be that while participants experienced the arm being there (and thus they reported that in the questionnaire), they were not as affected as in the uncovered conditions when the hand passed around because the arm was perceived to be under the cover. In the visuo-movement condition, GSR was high in both synchronous and asynchronous conditions. GSR may have been high in the asynchronous condition due to increased anxiety related to the inability to control the robot hand.

In addition, in all robot synchronous conditions of both experiments (visuo-tactile and visuo-movement) embodiment was very high (in a 7-point scale, average mean was rated above 4.5 in all conditions), also compared to previous studies which tested the RHI with other types of objects [2]. However, in Experiment 2, some participants complained that the gripper hand in the second experiment was reflecting the light and that it was not very clearly visible through the HMD: "With the gripper I did not feel that I can move or touch because the vision of the hand was not clear". This might explain why the gripper conditions obtained slightly lower means in the questionnaire than the other synchronous conditions.

As mentioned, the qualitative data resulting from the open-ended question in the first study and the interviews in the second study suggests that some participants experienced a stronger sense of embodiment when the illusion of embodiment was induced by visuo-movement synchronization while others experienced a stronger illusion when it was induced by visuo-tactile synchronization. Further experiments are needed to better understand these individual differences.

Finally, our findings are important in the arena of mediated embodiment, the technologically induced illusion of adopt- 
ing an artificial body in which one perceives to be located [35] in the sense that they contribute to explain how the illusion of embodiment in new technologies is created. In this regard, our results are in line with other findings made in virtual reality which suggest that avatar appearance does not necessarily need to be similar to the real appearance of the embodied subject $[15,16]$ to create sense of identification to its owner.

In addition, our results have important implications in the domain of robotics and extend the previous findings, which demonstrate that humans are able to embody highly-human resembling robotic and android limbs [12,14]. Our study demonstrates that humans can embody robotic limbs which are drastically different from a human limb in terms of shape, color, material, and texture. Humanoid robots that present human appearance but fail to attain it may generate eeriness, as shown in the Uncanny Valley hypothesis [36]. The Threat to distinctiveness hypothesis suggests that too much perceived similarity between robots and humans may trigger concerns about the negative impact of this technology because similarity blurs category boundaries, undermining human uniqueness [37]. Thus, studies on acceptance of nonhuman looking humanoids and its potential applications are important because these robots might be more appropriate for certain functions than highly human-resembling robots [38]. Finally, our results might also be relevant for the domain of prosthetics as they open new possibilities for limb prosthesis acceptance, in the sense that visuo-movement synchronization seem to be a more important factor contributing to integrate an external body limb as part of the own body than the visual resemblance to a human arm.

Acknowledgements This project has received funding from the European Union with the Marie Curie IOF Fellowship project HumRobCooperation under Grant agreement No. PIOF-CT-622764. It is also partially supported from the FP7 IP VERE No. 257695 and the Kakenhi 'houga' Grant 15616710 from the Japan Society for the Promotion of Science (JSPS). We specially thank Prof. E. Yoshida for his support in the ethical procedures and the interns at our laboratory in Japan who collaborated for the pretest or to appear in the pictures.

\section{Appendix 1}

Embodiment questionnaire for Study 1

From 1 (not at all) to 7 (very strongly), how strongly do you feel as if...

The arm you see was part of your body

The arm you see was in the location of your real arm

You could push an object with the arm you see

Embodiment questionnaire for Study 2

From 1 (not at all) to 7 (very strongly), it seems like...

You are looking directly at your own arm, rather than at a rubber/robot arm
The rubber/robot arm is part of your body

The rubber/robot arm is your arm

The rubber/robot arm belongs to you

The rubber/robot arm begins to resemble your real arm

The rubber/robot arm is in the location where your arm is

Your arm is in the location where the rubber/robot arm is

You could push an object with the arm you see

You could move the arm you see

Questions for the brief interview in Study 2

1. Describe a little bit the experience you just had.

2. From all the conditions you experienced, in which one the illusion that the arm you saw through the visor was strongest?

3. In general, was the sensation stronger in the synchronous or in the asynchronous conditions?

4. Was the sensation of seeing a human or a robot arm different, which one created a stronger illusion that the arm became your arm?

5. From all the (synch) robot arm conditions you experienced, in which one the illusion was stronger: the gripper or the arm with a blue cover? For the cover arm: the one in which we brushed your hand or the one in which you moved your hand and the robot hand moved?

6. Is there any sensation or anything else that you experienced which we did not ask you during the experiment that you would like to share with us? (e.g. anything that prevented you from having a stronger experience, etc.)

\section{Appendix 2}

Experimental conditions in Experiment 1 and 2 (Table 3).

Table 3 Experimental conditions by experiment

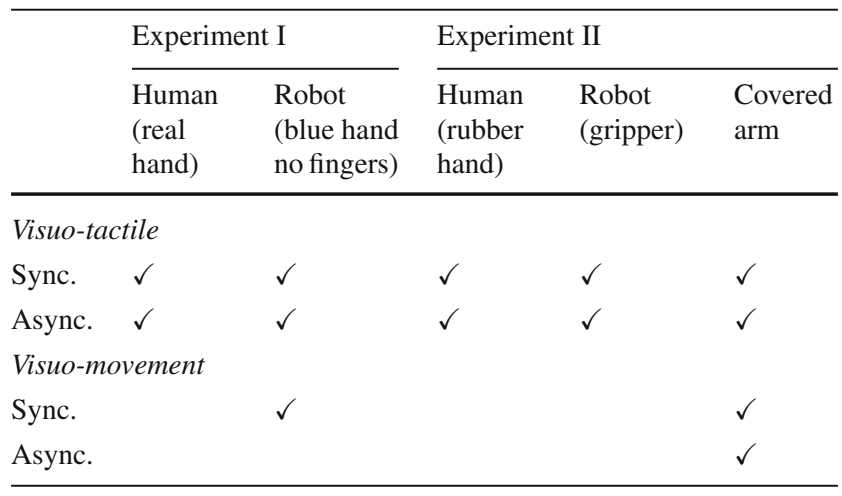

\section{Appendix 3}

Overall results for embodiment by condition in Experiment 1 and 2 (Table 4). 
Table 4 Embodiment scores by experimental condition for the two experiments

\begin{tabular}{|c|c|c|c|c|c|}
\hline & \multicolumn{2}{|l|}{ Experiment I } & \multicolumn{3}{|l|}{ Experiment II } \\
\hline & $\begin{array}{l}\text { Human (real hand) } \\
\text { M (SD) }\end{array}$ & $\begin{array}{l}\text { Robot (blue hand no fingers) } \\
\text { M (SD) }\end{array}$ & $\begin{array}{l}\text { Human (rubber hand) } \\
\text { M (SD) }\end{array}$ & $\begin{array}{l}\text { Robot (gripper) } \\
\text { M (SD) }\end{array}$ & $\begin{array}{l}\text { Covered arm } \\
\mathrm{M} \text { (SD) }\end{array}$ \\
\hline \multicolumn{6}{|c|}{ Visuo-tactile } \\
\hline Sync. & $5.11(1.06)$ & $5.33(1.04)$ & $5.13(1.35)$ & $4.56(1.41)$ & $4.97(1.33)$ \\
\hline Async. & $3.76(1.37)$ & $3.87(1.44)$ & $4.33(1.05)$ & 3.35 (1.6) & $3.59(1.56)$ \\
\hline \multicolumn{6}{|c|}{ Visuo-movement } \\
\hline Sync. & & $5.29(1.02)$ & & & $5.01(1.12)$ \\
\hline Async. & & & & & $2.76(1.78)$ \\
\hline
\end{tabular}

\section{References}

1. Botvinick M, Cohen J (1998) Rubber hands "feel" touch that eyes see. Nature 391:756. doi:10.1038/35784

2. Tsakiris M, Haggard P (2005) The rubber hand illusion revisited: visuotactile integration and self-attribution. J Exp Psychol Hum Percept Perform 31:80-91. doi:10.1037/0096-1523.31.1.80

3. Hohwy J, Paton B (2010) Explaining away the body: experiences of supernaturally caused touch and touch on non-hand objects within the rubber hand illusion. PLoS ONE 5:e9416. doi:10.1371/journal. pone. 0009416

4. Armel KC, Ramachandran VS (2003) Projecting sensations to external objects: evidence from skin conductance response. Proc Biol Sci 270:1499-506. doi:10.1098/rspb.2003.2364

5. Maselli A, Slater M (2013) The building blocks of the full body ownership illusion. Front Hum Neurosci 7:83. doi:10.3389/fnhum. 2013.00083

6. Holmes NP, Snijders HJ, Spence C (2006) Reaching with alien limbs: visual exposure to prosthetic hands in a mirror biases proprioception without accompanying illusions of ownership. Percept Psychophys 68:685-701. doi:10.3758/BF03208768

7. Longo MR, Schüür F, Kammers MPM et al (2009) Self awareness and the body image. Acta Psychol (Amst) 132:166-172. doi:10. 1016/j.actpsy.2009.02.003

8. Pavani F, Zampini M (2007) The role of hand size in the fake-hand illusion paradigm. Perception 36:1547-1554. doi:10.1068/p5853

9. Ehrsson HH, Spence C, Passingham RE (2004) That's my hand! Activity in premotor cortex reflects feeling of ownership of a limb. Science 305:875-877. doi:10.1126/science.1097011

10. Costantini M, Haggard P (2007) The rubber hand illusion: sensitivity and reference frame for body ownership. Conscious Cogn 16:229-240. doi:10.1016/j.concog.2007.01.001

11. Lloyd DM (2007) Spatial limits on referred touch to an alien limb may reflect boundaries of visuo-tactile peripersonal space surrounding the hand. Brain Cogn 64:104-109. doi:10.1016/j.bandc. 2006.09.013

12. Romano D, Caffa E, Hernandez-Arieta A et al (2015) The robot hand illusion: Inducing proprioceptive drift through visuomotor congruency. Neuropsychologia 70:414-420. doi:10.1016/j. neuropsychologia.2014.10.033

13. Hellman RB, Chang E, Tanner J et al (2015) A robot hand testbed designed for enhancing embodiment and functional neurorehabilitation of body schema in subjects with upper limb impairment or loss. Front Hum Neurosci 9:1-10. doi:10.3389/fnhum.2015.00026

14. Alimardani M, Nishio S, Ishiguro H (2013) Humanlike robot hands controlled by brain activity arouse illusion of ownership in operators. Sci Rep 3:2396. doi:10.1038/srep02396
15. Aymerich-Franch L (2012) Can we identify with a block ? Identification with non-anthropomorphic avatars in virtual reality games. In: Proc. Int. Soc. Presence Res. Annu. Conf

16. Aymerich-Franch L (2010) Presence and emotions in playing a group game in a virtual environment: the influence of body participation. Cyberpsychol Behav Soc Netw 13:649-654. doi:10.1089/ cyber.2009.0412

17. Aymerich-Franch L, Kizilcec RF, Bailenson JN (2014) The relationship between virtual self similarity and social anxiety. Front Hum Neurosci 8:1-10. doi:10.3389/fnhum.2014.00944

18. Aymerich-Franch L, Petit D, Ganesh G, Kheddar A (2015) Embodiment of a humanoid robot is preserved during partial and delayed control. In: 2015 IEEE Int. Work. Adv. Robot. its Soc. Impacts

19. Petit D, Gergondet P, Cherubini A, Kheddar A (2015) An integrated framework for humanoid embodiment with a BCI. In: IEEE Int. Conf. Robot. Autom

20. Kaneko K et al (2004) Humanoid robot HRP-2. In: IEEE Int. Conf. Robot. Autom

21. Mansard N, Stasse O, Evrard P, Kheddar A, (2009) A versatile generalized inverted kinematics implementation for collaborative working humanoid robots: the stack of tasks. In: 2009 Int. Conf. Adv. Robot

22. Kilteni K, Groten R, Slater M (2012) The sense of embodiment in virtual reality. Presence Teleoper Virtual Environ 21:373-387. doi:10.1162/PRES_a_00124

23. Longo MR, Schüür F, Kammers MPM et al (2008) What is embodiment? a psychometric approach. Cognition 107:978-998. doi:10. 1016/j.cognition.2007.12.004

24. Blanke O, Metzinger T (2009) Full-body illusions and minimal phenomenal selfhood. Trends Cogn Sci 13:7-13. doi:10.1016/j. tics.2008.10.003

25. Sackett DL (1979) Bias in analytic research. J Chronic Dis 32:5163. doi:10.1016/0021-9681(79)90012-2

26. Connor KM, Kobak KA, Churchill LE et al (2001) Mini-SPIN: a brief screening assessment for generalized social anxiety disorder. Depress Anxiety 14:137-140

27. Seeley-Wait E, Abbott MJ, Rapee RM (2009) Psychometric properties of the mini-social phobia inventory. Prim Care Companion J Clin Psychiatry 11:231-236. doi:10.4088/PCC.07m00576

28. Aymerich-Franch L, Petit D, Ganesh G, Kheddar A (2016) The second me: seeing the real body during humanoid robot embodiment produces an illusion of bi-location. Conscious Cogn 46:99-109. doi:10.1016/j.concog.2016.09.017

29. Sanchez-Vives MV, Spanlang B, Frisoli A et al (2010) Virtual hand illusion induced by visuomotor correlations. PLoS ONE 5:e10381. doi:10.1371/journal.pone.0010381

30. Aymerich-Franch L, Ganesh G (2015) The role of functionality in the body model for self-attribution. Neurosci Res. doi:10.1016/j. neures.2015.11.001 
31. Ehrsson H (2012) The concept of body ownership and its relation to multisensory integration. In: Stein BE (ed) The new handb. multisensory process. Cambridge, MA: Mit Press, pp 775-792

32. MacKenzie IS (2013) Human-computer interaction: an empirical research perspective. Morgan Kaufmann, Waltham

33. Kantowitz BH, Roediger HL III, Elmes DG (2015) Experimental psychology. Cengage Learning, Stamford, CT, USA

34. Greenwald AG (1976) Within-subjects designs: to use or not to use? Psychol Bull 83:314-320. doi:10.1037/0033-2909.83.2.314

35. Aymerich-Franch L Mediated embodiment in new communication technologies. In: Khosrow-Pour M (ed) Encyclopedia of information science and technology, 4th edn. Hershey, IGI Global (in press)

36. Mori M (1970) The uncanny valley. Energy 7:33-35. doi:10.1162/ pres.16.4.337

37. Ferrari F, Paladino MP, Jetten J (2016) Blurring human-machine distinctions: anthropomorphic appearance in social robots as a threat to human distinctiveness. Int J Soc Robot 1-16. doi:10.1007/ s12369-016-0338-y

38. Złotowski J, Proudfoot D, Yogeeswaran K, Bartneck C (2014) Anthropomorphism: opportunities and challenges in humanrobot interaction. Int J Soc Robot 7:347-360. doi:10.1007/ s12369-014-0267-6

Laura Aymerich-Franch received a Ph.D. cum laude on Audiovisual Communication by Universitat Autònoma de Barcelona. She is currently a Marie Curie IOF fellow at The Experimental Virtual Environments (EVENT) Lab for Neuroscience and Technology at the University of Barcelona (return phase). She completed the outgoing phase of the Marie Curie fellowship at the CNRS-AIST Joint Robotics Laboratory (JRL) in AIST, Japan (2014-2016). Previously, she was a Fulbright postdoctoral researcher at Virtual Human Interaction Lab, Stanford University (2012-2014). Her area of expertise is Media Psychology applied to advanced communication technologies. Currently, she studies the psychological aspects of mediated embodiment in humanoid robots and virtual reality.

Damien Petit received his M.Eng. degree and the M.Sc. degree in robotics and computer vision from the Télécom Physique Strasbourg, France, in 2010. He received his Ph.D. in Robotics from the University of Montpellier, France, in December 2015; his research was conducted in large part at the CNRS-AIST Joint Robotics Laboratory in Tsukuba, Japan, and at the Interactive Digital Human group of LIRMM, at Montpellier, in the frame of the European Union FP7 Virtual Embodiment and Robotic re-Embodiment (VERE) project. His research interests include humanoid robotics, robotic embodiment, robot-human interactions and thought-based control of humanoid.
Gowrishankar Ganesh received his Bachelor of Engineering (firstclass, Hons.) degree from the Delhi College of Engineering, India, in 2002 and his Master of Engineering from the National University of Singapore, in 2005, both in Mechanical Engineering. He received his Ph.D. in Bioengineering from Imperial College London, UK, in 2010. He worked as a Researcher in Human Motor Control in the Advanced Telecommunication Research (ATR), Kyoto, Japan, from 2004 and through his Ph.D. Following his Ph.D., he worked at the National Institute of Information and Communications Technology (NICT) as a Specialist Researcher in Motor Neuroscience and Robotics till December 2013. Since January 2014, he has joined as a CR1 Researcher at the Centre National de la Recherche Scientifique (CNRS), and is currently located at the CNRS-AIST Joint Robotics Laboratory (JRL) in Tsukuba, Japan. He is a visiting researcher at the Centre for Information and Neural Networks (CINET) in Osaka, ATR in Kyoto and the Laboratoire d'Informatique, de Robotique et de Microlectronique de Montpellier (LIRMM) in Montpellier. His research interests include human sensorimotor control and learning, robot control, social neuroscience and robot-human interactions.

Abderrahmane Kheddar received the BS in Computer Science degree from the Institut National d'Informatique (ESI), Algiers, the M.Sc. and Ph.D. degree in robotics, both from the University of Pierre et Marie Curie, Paris. He is presently Directeur de Recherche at CNRS and the Director of the CNRS-AIST Joint Robotic Laboratory (JRL), UMI3218/RL, Tsukuba, Japan. He is also leading the Interactive Digital Humans (IDH) team at CNRS-University of Montpellier LIRMM, France. His research interests include haptics, humanoids and thoughtbased control using brain machine interfaces. He is a founding member of the IEEE/RAS chapter on haptics (acting also as a senior advisor), the co-chair and co-founding member of the IEEE/RAS Technical committee on model-based optimization. He is presently Editor of the IEEE Transactions on Robotics, the Journal of Intelligent and Robotic Systems, and Frontiers in Bionics; among the founders of the IEEE Transactions on Haptics he served in its editorial board from 2007 to 2010 and served as an associate editor in the MIT Press Presence journal. He is an IEEE Senior Member, Member of the steering committee of the IEEE Brain Initiative, Titular member of the National Academy of Technology of France, and Knight of the National Order of the Merit. 